\title{
Physical mechanism for origin of streamwise vortices in mode $A$ of a square-section cylinder
}

\author{
L. M. $\operatorname{Lin}^{1} \cdot$ S. Y. Shi ${ }^{1} \cdot$ Y. X. $\mathrm{Wu}^{1,2}$
}

Received: 4 June 2018 / Revised: 31 August 2018 / Accepted: 20 September 2018

(c) The Chinese Society of Theoretical and Applied Mechanics; Institute of Mechanics, Chinese Academy of Sciences and Springer-Verlag GmbH Germany, part of Springer Nature 2018

\begin{abstract}
In the present paper, physical mechanism responsible for origin of streamwise vortices in mode A appeared in the threedimensional (3-D) wake transition of a square-section cylinder is investigated. Direct numerical simulations at a Reynolds number of 180 firstly show that such streamwise vorticity is not originated from lateral surfaces. Then through the analysis of local flow field in the immediate neighborhood of rear surface, based on the theory of vortex-induced vortex, a new physical mechanism is identified. At first, the vertical vorticity on rear surface is generated by the intrinsic three-dimensional instability with the same instability wavelength of mode A. Then the streamwise vorticity at a specific sign is induced by such vertical vorticity, convected and concentrated in the shear layers. Finally, streamwise vortices are formed and shed with alternatively shedding spanwise vortices in the near wake. Moreover, the effect of induced spanwise vorticity on original two-dimensional (2-D) spanwise vorticity is also presented in detail.
\end{abstract}

Keywords Mode A $\cdot$ Streamwise vortices $\cdot$ Wake $\cdot$ Square cylinder $\cdot$ Vortex-induced vortex

\section{Introduction}

Flow past a bluff body is a classical problem in fluid mechanics, studied extensively in the literature [1-11] and applied for engineering, such as marine risers. Particularly in the threedimensional (3-D) wake transition for flow past a circular- or square-section cylinder, two 3-D instability modes, referred as modes $\mathrm{A}$ and $\mathrm{B}$, have been discovered in experiments and direct numerical simulations. However, there still has a problem unclearly illustrated in previous works, i.e. , the origin of streamwise vortices in mode A. It is very important and related to different physical mechanism in generation of streamwise vortices appeared in mode A.

First of all, let us review the phenomenon in mode A 3-D instability, as shown in Fig. 1 for direct numerical simulations of flows past cylinders with circular and square cross-sections $[6,9,11]$. The Reynolds number $R e$ is based on the free stream

S. Y. Shi

shishiying@imech.ac.cn

1 Key Laboratory for Mechanics in Fluid Solid Coupling Systems, Institute of Mechanics, Chinese Academy of Sciences, Beijing 100190, China

2 School of Engineering Sciences, University of Chinese Academy of Sciences, Beijing 100049, China velocity and base height of cylinder. In the flow past a circular cylinder, typically as shown in Fig. 1a, the near wake can be described by the large-scale streamwise vortices and spanwise vortices alternatively shed from the upper and lower shear layers. The spanwise vortices are obviously undulated across the span. Another feature is the streamwise vorticity with opposite signs in a staggered arrangement from one braid region to the next one. Similar phenomena are also observed in the wake of a square cylinder, as shown in Fig. 1c.

As for the appearance of streamwise vortices with specific signs, two basic mechanisms have been proposed in recent three decades in previous works $[1-3,8,10,12,13]$. In the first mechanism, the streamwise vorticity in mode $\mathrm{A}$ is considered to be originated from that on cylinder surfaces. It is already known that the vorticity is firstly generated on the solid walls due to the viscous forces if the incoming flow is uniform and incompressible and the body forces are potential [14]. Then initial stage of a 3-D vortex structure in a two-dimensional (2-D) boundary layer separation flow is investigated $[12,13]$. The wavy separation line on surface of a circular cylinder is observed in experiments, as shown in Fig. 2a. Correspondingly, the laminar boundary layer is wavily separated from the side of a separated region. It is clearly shown in Fig. 2a 

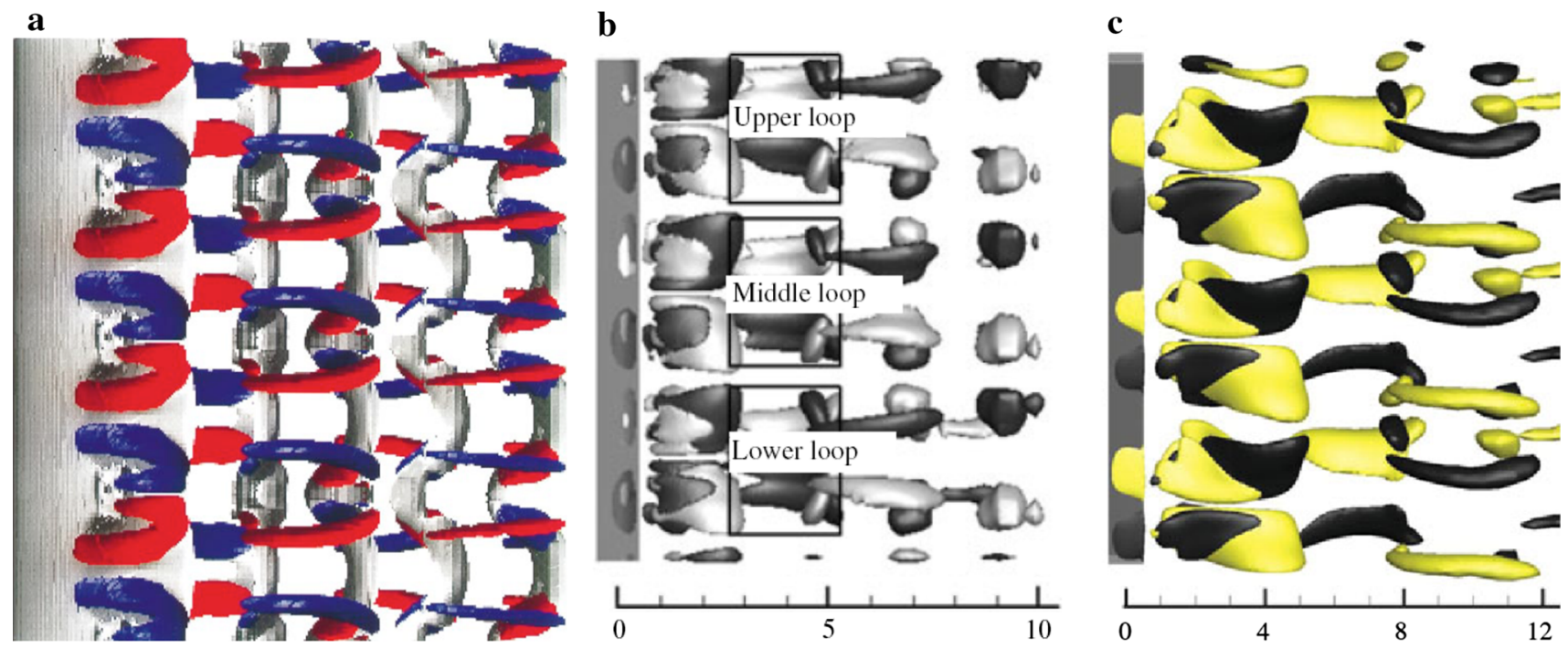

Fig. 1 In the near wake, a iso-surfaces of streamwise vorticity, $\omega_{x}= \pm 0.75$ (red/blue), and spanwise vorticity, $\omega_{z} \pm 1$ (silver), at Reynolds number $R e=195$ for a circular cylinder [6], $\mathbf{b}$ iso-surfaces of $\omega_{x} \pm 0.5$ (dark grey/light grey), at dimensionless time $t=3050$ and $R e=240$ for a circular cylinder [9], $\mathbf{c}$ iso-surfaces of $\omega_{x} \pm 0.6$ (black/yellow), at $t=140$ and $R e=200$ for a square cylinder [11]. The flow is from the left to the right past the cylinder on the left
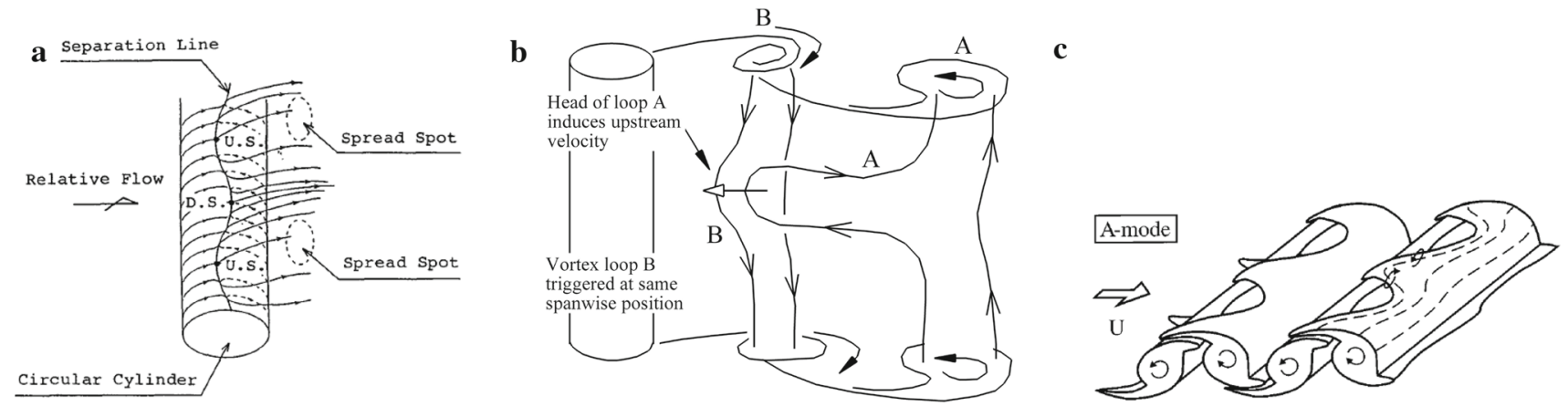

Fig. 2 In the first mechanism, a explanation sketch of the 3-D separation from a circular cylinder [12,13], where symbol "•" denotes the singular separation point with zero shear stress at wall, U.S. and D.S. denote upstream and downstream singular separation points, respectively. In the second mechanism, b schematics of the formation process of vortex loops in mode A [3], where segments of the primary vortex cores are pulled out of the primary structure as it is shed, and form an array of vortex loops, $\mathbf{c}$ schematics of the primary and secondary vortices in mode A illustrated by the vortex tongues wrapped around the Kármán vortex roller [2]

that the originally parallel surface streamlines in the upstream region, illustrated by solid lines, become 3-D after leaving the surface, and the distances between neighboring streamlines become either wider or narrower. This indicates that the spanwise velocity is appeared near such wavy separation line, as a direct result of intrinsic 3-D instability. Consequently, the streamwise vortices are generated behind those wavy separated regions, just as shown by $\omega_{x}$ on cylinder surfaces in Fig. $1 \mathrm{~b}, \mathrm{c}$. Moreover, there exists a spanwise correlation between the 3-D features of the separation and the 3-D wake structure in the range of Reynolds number from 170 to 1340, such as the normalized correlation length about 9 at $R e=170$. At least in present mechanism, the streamwise vortices are initially originated near the cylinder surface, particularly the separated region.
However, as for the second physical mechanism, it is responsible for the appearance of streamwise vortices in the shear layers and near wake of a cylinder, as well as the resultant wavy spanwise vortices. In most literature $[1,3,4,8]$, it is believed that such mechanism is attributed to the deformation of primary vortices due to an elliptic instability of the primary vortex cores during the process of shedding, causing a spanwise waviness, and coupled effect of BiotSavart induction through the wake visualizations. As shown in Fig. 2b, the subsequent growth of vortex loops is due to a feedback mechanism from one primary vortex to the next. These vortex loops are self-sustaining at the same spanwise location. A previously formed vortex loop A convects close to the shedding primary vortex $\mathrm{B}$. The induced velocity due to the head of the loop A will retard a segment of the vortex 
B at the same spanwise position, by Biot-Savart induction. From this segment, a vortex loop will be drawn out of the primary structure. The process will continue from one vortex to the next in a self-sustaining mode. From the sides of the loops, streamwise vortices will roll up, in a process which naturally causes an out-of-phase symmetry of the streamwise vortex array. Similarly, based on the spatio-temporal vortex structures observed experimentally and on analysis of the 2$\mathrm{D}$ velocity field, the model of vortex tongues in the vortex sheet is proposed [2]. As shown in Fig. 2c, vortex sheets in the shape of "tongues" are pulled out of a Kármán vortex tube and wrapped around the succeeding one. Each tongue is composed of vortex lines (dashed lines in Fig. 2c), which are stretched in the streamwise direction and form a pair of secondary vortices, i.e. the fragmented patches with streamwise vorticity. And the mode A is likely to be the result of an instability of the braid region between the primary Kármán vortices due to centrifugal forces. Recently through computational analysis [10], such mechanism is also to be originated from an axial stretching of the upstream perturbed vroticity, existing on the braids, due to the strain field created by the spanwise vortices which evolve under a shear instability of the wake when viscous effects are small.

Furthermore, an obvious confusion is identified when these two mechanisms are used to explain the origin of streamwise vortices. For example, as shown in Fig. 1b, the lower spanwise position is taken into account in the lower loop with alternatively shedding streamwise vortices. The streamwise vorticity at a positive sign (denoted by $\left.+\left|\omega_{x}\right|\right)$ is distributed on cylinder surface $(0<x<0.5)$, but the sign of streamwise vorticity in the same shedding side of mode $\mathrm{A}(5<x<8)$ is negative (denoted by $\left.-\left|\omega_{x}\right|\right)$ at the same spanwise location. Meanwhile, the streamwise vorticities with positive and negative signs are all coexisted in the same side of shear layer $(1<x<3)$. That is to say, the sign of streamwise vorticity in the near wake is just opposite to that on cylinder surface. And another feature is $+\left|\omega_{x}\right|$ obviously less than $-\left|\omega_{x}\right|$ with larger iso-surface region in the shear layer $(1<x<3)$. Similar phenomena are also appeared in the wake flow of a square cylinder, as shown in Fig. 1c. Based on the above analysis in Fig. 1b, it is certainly confirmed that the first mechanism is not enough to explain such feature because of weak intensity of $+\left|\omega_{x}\right|$, and the second mechanism also can not provide a reasonable answer for the coexistence of $\omega_{x}$ with opposite signs in the same shear layer, even the appearance of $\omega_{x}$ on cylinder surface.

Therefore, it is believed that there must exist a new physical mechanism to explain the appearance of streamwise vorticity with a specific sign in the shear layer, just opposite to that on cylinder surface. Recently, a new phenomenon about the specific sign combination of different vorticity components is found out in numerical simulations of the near wake of peak-perforated conic shroud [15]. And it is confirmed by the theory of vortex-induced vortex (VIVor) [16], which illustrates the resultant vorticity and velocity fields induced by introducing a specific vortex (or vortex pair) under a particular boundary condition. Particularly, the first sign law, the intrinsic physical relationship between $\omega_{x}$ and $\omega_{y}$, may be adopted in further analysis of origin of streamwise vortices in mode A. On the other hand, effect of the vertical component of vorticity, $\omega_{y}$, is usually ignored or underestimated, even less reported in previous works [3,4,9,11]. Actually, it plays a key role in the wavy formation of spanwise vortices distorted towards and outwards the cylinder, such as $\Omega$-type vortices, even the complete suppression of Kármán vortices [15].

In present paper, the main aim is about to provide a new explanation based on numerical simulations of flow past a square cylinder and the VIVor theory. It should be emphasized here that the reason for adopting the cylinder with square cross-section is mainly attributed to specific distributions of streamwise and vertical vorticities on cylinder surfaces. As for the square cylinder, only $\omega_{x}$ is generated on lateral surfaces, but $\omega_{y}$ is generated on rear surface. However as for the circular cylinder, both $\omega_{x}$ and $\omega_{y}$ are all appeared on curved surfaces. Resultantly, different effects of streamwise and vertical vorticities can be clearly identified in the wake of the square cylinder. In Sect. 2, the physical model, including computational method and boundary conditions, is introduced firstly in brief. Then the vorticity on cylinder surfaces and in the near wake is presented numerically and analyzed theoretically in detail in Sect. 3. Finally, the conclusion is made in Sect. 4.

\section{Physical model}

An incompressible flow past the square-section cylinder is taken into account, as shown in Fig. 3a. Among them, the Cartesian coordinate system $(x, y, z)$ is established, where the $x$-axis (streamwise) is aligned with the incoming free stream, the $z$-axis (spanwise) is parallel to the cylinder span, while the $y$-axis (vertical) is normal to the $(x, z)$ plane. The density $\rho$ and kinematic viscosity $v$ of the fluid are constant.

The continuity and Navier-Stokes equations in dimensionless forms are written out by

$$
\begin{aligned}
& \nabla \cdot \boldsymbol{u}=0, \\
& \frac{\partial \boldsymbol{u}}{\partial t}+(\boldsymbol{u} \cdot \nabla) \boldsymbol{u}=-\nabla p+\frac{1}{R e} \nabla^{2} \boldsymbol{u},
\end{aligned}
$$

where $\boldsymbol{u}$ is the velocity vector $(u, v, w), t$ is non-dimensional time, $\nabla$ is the gradient operator, $p$ is the static pressure, $R e$ is the Reynolds number defined as $U_{\infty} D / \nu$, where $U_{\infty}$ is the velocity of free stream and $D$ is the base height of square cylinder. Velocities are characterized by $U_{\infty}$ and lengthes 


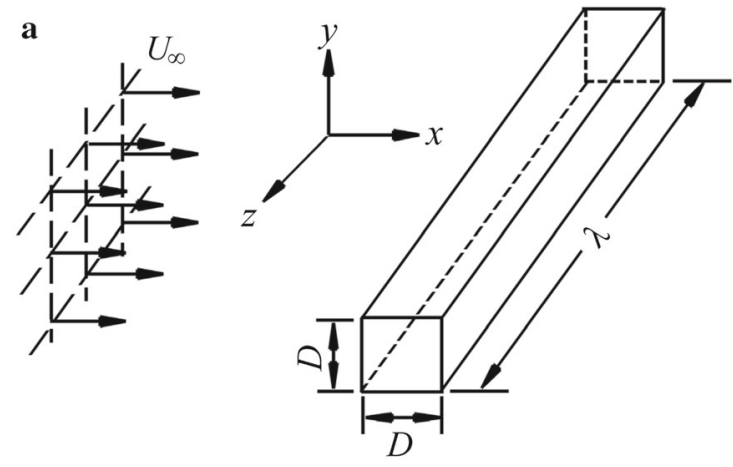

b

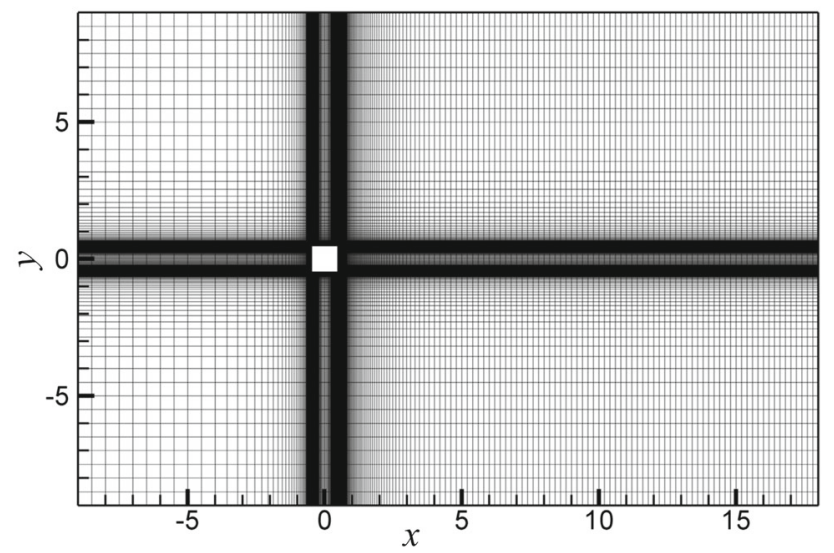

Fig. 3 a Sketch of flow past a square-section cylinder. b Nondimensional computational domain and mesh distribution in the $(x, y)$ plane

by $D$. In present paper, the computational Reynolds number is 180 in order to capture the early stage, beyond the critical Reynolds number of 161 at which the mode A 3-D instability is appeared by the Floquet analysis [17].

Correspondingly the appropriate boundary conditions are employed as follows. As for velocity, the uniform free stream, $u=U_{\infty}$ and $v=w=0$, is adopted at the inlet of computational domain. The simple convective outflow, $\frac{\partial \boldsymbol{u}}{\partial t}+U_{\mathrm{c}} \frac{\partial \boldsymbol{u}}{\partial x}=$ 0 with the convective velocity $U_{\mathrm{c}}=1$, is applied at the outlet. On both lateral sides of computational domain in the $y$-axis, free slip boundary conditions, $\frac{\partial u}{\partial y}=v=\frac{\partial w}{\partial y}=0$, are employed. And non-slip boundary conditions, $\boldsymbol{u}=\mathbf{0}$, are used on cylinder surfaces. While for pressure, the compatible pressure boundary conditions, the normal component of momentum equation, are implicitly computed at all boundaries of computational domain. And the reference pressure $p_{\infty}$ is zero at the center of inlet $y=0$ to avoid the numerical drift.

In spacial discretization, the finite difference method is adopted with the central difference scheme in the $(x, y)$ plane and the spectral method with Fourier function is applied along the span based on the assumption that the flow is periodic across the span. As for the time scheme for unsteady and convective terms, and so on, the second-order splitting method is adopted [18].

The dimensionless computational domain in the $(x, y)$ plane is $27 \times 18(x \times y)$, as shown in Fig. 3b. And the nondimensional spanwise computational length $\lambda$ in Fig. 3a is assumed to be one spanwise wavelength of mode A 3-D instability, i.e., $\lambda=6$, just to capture main features in specific distributions of vorticity in the near wake, as a pure mode $\mathrm{A}$ without effect of vortex dislocation $[3,4,6,9,11]$. The finest grids with non-dimensional spacing about $10^{-3}$ are clustered near the cylinder and the coarsest grids with spacing about 0.1 are distributed away from the cylinder, as shown in Fig. $3 \mathrm{~b}$.

Total computational Fourier mode for mode A is 32 . The $n$th wavenumber $\beta_{n}$ is defined as $2 \pi n / \lambda$ in spectral space. In Eq. (2), the spanwise viscous item, $\frac{1}{R e} \frac{\partial^{2} u}{\partial z^{2}}$, can be expressed by a sum of Fourier modes, $\frac{1}{R e} \sum \beta_{n}^{2} \hat{\boldsymbol{u}}_{n} \mathrm{e}^{\mathrm{i} \beta_{n} z}$, where $\hat{\boldsymbol{u}}_{n}(t, x, y)$ is the $n$-th spanwise Fourier mode of $\boldsymbol{u}(t, x, y, z)$. Therefore, viscous dissipation becomes important at wavenumbers $\beta_{D} \sim \operatorname{Re}^{\frac{1}{2}}$. And at wavenumbers $\beta_{n}>\beta_{D}$ the momentum equations are dominated by viscous forces [5,6]. These high-wavenumber modes contribute little to the dynamics of the flow at large scales because their energy is rapidly dissipated by viscosity. For an adequate description of the dynamics in a system with a given spanwise dimension $\lambda$, we only need a finite set of $N$ Fourier modes to cover the range of scales from $\beta_{0}=0$ (the mean flow) to $\beta_{D}=2 \pi N / \lambda \sim \operatorname{Re}^{\frac{1}{2}}$, or $N \sim \lambda \operatorname{Re}^{\frac{1}{2}} /(2 \pi)$. Hereby, we just investigate the 3-D flow dynamics with spanwise periodicity at the smallest periodic resolution of $N=32$ at $R e=180$ in present paper, considering to eliminate the aliasing error in computing non-linear convective terms.

Verification of computational codes is mainly carried out at $R e=100$. And dependence study for mesh and computational domain can be referenced in the work of Lin [19]. For example, the Strouhal number $S t$, defined as $f D / U_{\infty}(f$ is the vortex-shedding frequency), is obtained to be 0.149 , agreed well with 0.146 in experiments [20].

\section{Results and discussions}

\subsection{Mode A with specific distributions of vorticity components}

The wake of a square-section cylinder at a Reynolds number of 180, as shown in Fig. 4a, can be described by the alternatively shedding П-type vortices. The moment of such vortex-shedding pattern is picked up after the wake flow is steady and periodic along the time, illustrated by periodical variations of drag coefficient $C_{D}$, its pressure contribution $C_{D p}$ and lift coefficient $C_{L}$ in Fig. 4b. In such П-type vortex, the head line, "--", denotes the spanwise vortex, while 


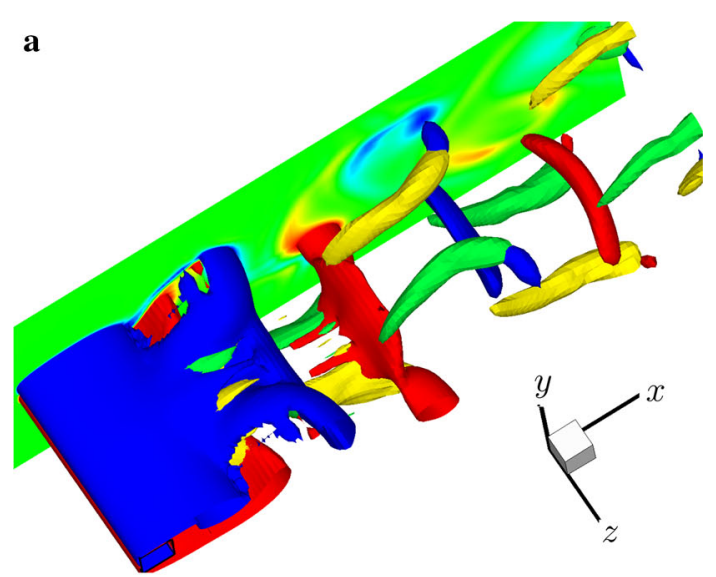

b
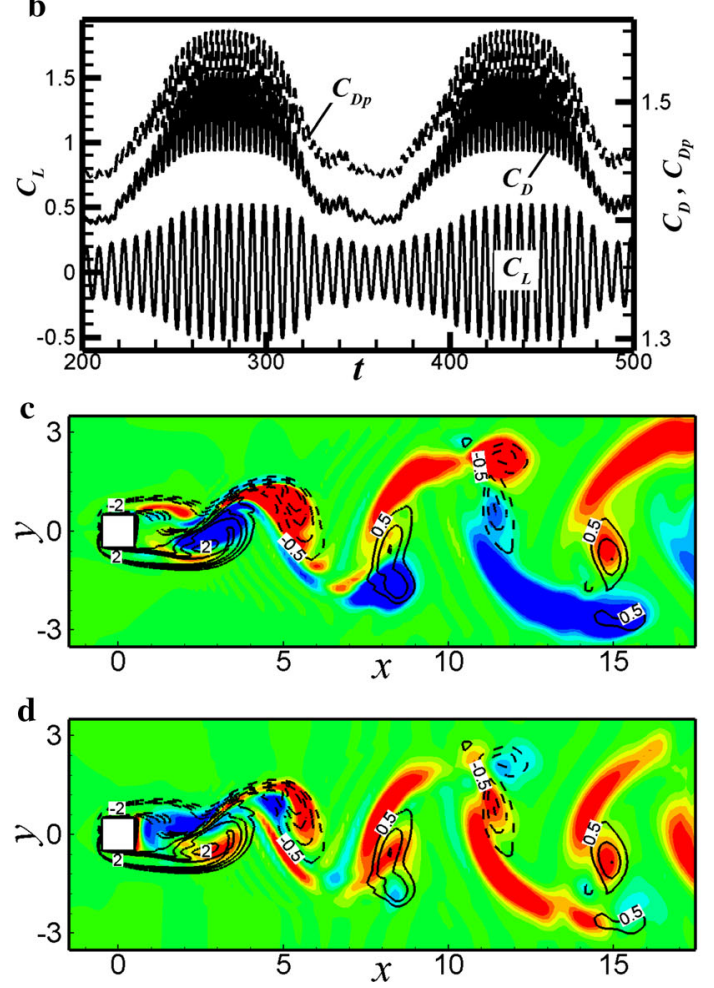

Fig. 4 In the wake of a square cylinder at $t=340, \lambda=6$ and $R e=180$, a iso-surfaces of $\omega_{x} \pm 0.8$ (yellow/green) and $\omega_{z} \pm 1$ (red/blue) with sectional contours of $\omega_{z}$ at $z=0$ and $\mathbf{b}$ time histories of drag coefficient $C_{D}$, its pressure contribution $C_{D p}$ and lift coefficient $C_{L}$. Contours of c $\omega_{x}$ and $\mathbf{d} \omega_{y}$ at $z=\frac{1}{4} \lambda$, where red and blue colors, as well as solid and dashed lines for contours of $\omega_{z}$, denote positive and negative values, respectively

two legs, "I |", denote streamwise and/or vertical vortex pairs shed with this spanwise vortex and elongated or stretched by the upstream spanwise vortex of opposite sign [15,19]. It is consistent with the physical description in previous works $[3,4]$.

Meanwhile, there are specific signs of additional components of vorticity, i.e. $\omega_{x}$ and $\omega_{y}$, distributed in the near wake. Typically at non-dimensional spanwise position $z=\frac{1}{4} \lambda$, it can be shown that the streamwise vortices with positive and
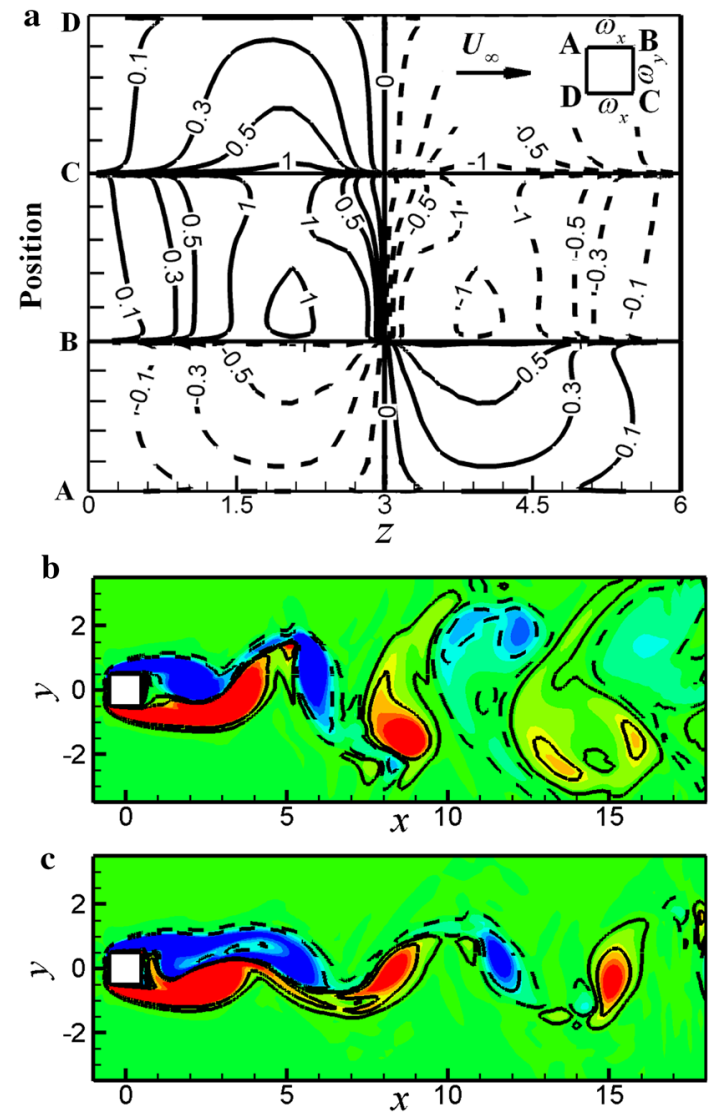

Fig. 5 a Contours of $\omega_{x}$ on lateral surfaces $\mathrm{AB}$ and $\mathrm{CD}$ and $\omega_{y}$ on rear surface $\mathrm{BC}$, and contours of $\omega_{z}$ at $\mathbf{b} z=0$ and $\mathbf{c} z=\frac{1}{2} \lambda$, where red and blue colors, as well as solid and dashed lines only at $\omega_{z} \pm 0.1$ and \pm 0.5 in sub-figure $\mathbf{b}$ and $\mathbf{c}$, denote positive and negative values, respectively

negative vorticity signs are shed from the upper and lower shear layers, respectively, as shown in Fig. 4c. While the vertical vortices only with a positive vorticity sign are shed from both shear layers at same spanwise position, as shown in Fig. 4d. Particularly in present circumstances, the streamwise vorticity with a positive sign is originally appeared and gradually concentrated in the upper shear layer, and finally shed with the negative spanwise vorticity.

Now let us put the focus on vorticity distributions on cylinder surfaces, as shown in Fig. 5a. The sign of streamwise vorticity at $z=\frac{1}{4} \lambda$ on the upper surface $A B$ is negative, just opposite to that in the upper shear layer and near wake, as well as that on and in the lower side. Such phenomenon is exactly agreed with the previous work $[9,11]$ in Fig. 1b, c. Interestingly, it is also found out that only streamwise vorticity with a positive sign is appeared in the upper shear layer $(0.5<x<2)$ at present $R e=180$ in Fig. 4c, dislike streamwise vorticities with opposite signs coexisted in region of $1<x<3$ at $\operatorname{Re}=240$ in Fig. $1 \mathrm{~b}$ or $1<x<5$ at $R e=200$ in Fig. 1c. This phenomenon shows that the first mechanism, the streamwise vorticity originated from the sur- 

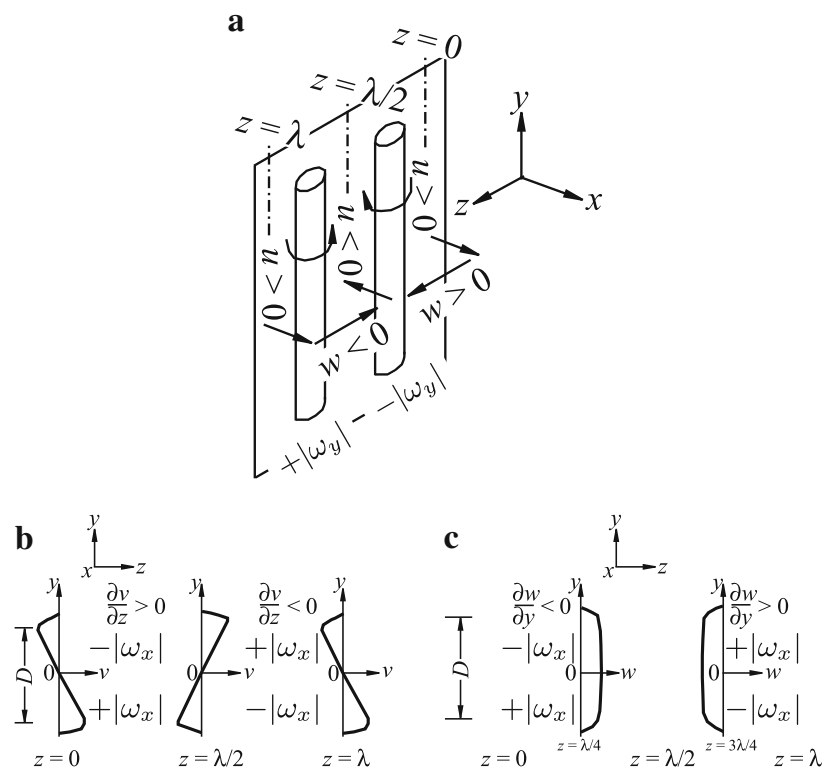

Fig. 6 a Schematics of introduced vertical vortex pairs $\left(-\left|\omega_{y}\right|,+\left|\omega_{y}\right|\right)$ and induced velocity field $(u, w)$ varied along the $z$-axis on rear surface of the straight square cylinder, and sketches of qualitative distributions of induced velocities, $\mathbf{b} v$ and $\mathbf{c} w$, and induced streamwise vorticity, $\omega_{x}$, near the rear surface of a square cylinder with the finite height $D$, just denoted by the solid line with two filled arrowheads attached on its both ends

face separated region, is really existed on lateral surfaces but suppressed just behind the body at lower Reynolds number because of larger viscous forces. At least, it is certainly confirmed too that such mechanism is not the dominant physical mechanism responsible for the origin of streamwise vortices in mode $\mathrm{A}$.

\subsection{Analysis by means of the VIVor theory}

Here we mainly concern the new physical mechanism originated from the rear surface, according to the VIVor theory. A simple case just as shown in Fig. 6a is adopted, according to the specific distribution of vertical vorticity on rear surface in Fig. 5a. The local flow in the immediate neighborhood of flat rear surface of the straight square-section cylinder where the viscous forces are dominant is further analyzed. On the other hand, Kelvin's circulation theory [14] indicates that the circulation and the vorticity flux for the same and single vortex with a specific sign are always invariable. The inertial forces have no effect on the evolution of vorticity sign and therefore can be neglected. Then the introduced vertical vortex pairs are assumed to be uniformly distributed on rear surface along the vertical direction. Similar to streamwise vorticity appeared in the local separated region due to the intrinsic 3-D instability, such introduced vertical vortex pairs are also resulted from the intrinsic 3-D instability near the rear surface with present instability wave- length. In present circumstances that the inertial forces are neglected, such local flow field with vorticity $\left(\omega_{x}, \omega_{y}, \omega_{z}\right)$ can be decomposed into two parts: the original 2-D wake flow past the cylinder with only spanwise vorticity $\Omega_{z}(x, y)$, and the induced 3-D flow fields in present local region with induced vorticity $\left(\omega_{x}, \omega_{y}, \delta \omega_{z}\right)$, in which $\omega_{z}=\Omega_{z}+\delta \omega_{z}$.

Now as for the latter, the local Cartesian coordinate system $(x, y, z)$ is established, as shown in Fig. 6a, just consistent with the system in Fig. 3a. Under consideration of conservative body forces, the incompressible continuity and momentum equations in the non-dimensional form can be written by

$$
\begin{aligned}
& \nabla \cdot \boldsymbol{u}=0, \\
& \nabla p=\frac{1}{R e} \nabla^{2} \boldsymbol{u} .
\end{aligned}
$$

And the non-slip boundary conditions, $u=v=w=0$, at walls are employed. The first boundary case [16], in which the introduced vorticity is generated on cylinder surfaces with the amplitude of $A_{\omega_{y}}$, similar to that in Fig. 5a, is adopted.

Therefore in above local flow region, exact solutions for the induced velocity and vorticity fields can be obtained [16]. Firstly, the induced velocity field is assumed in the following forms

$$
\begin{aligned}
& u(x, z)=A_{u}(x) \cos (\beta z), \\
& v(x, y, z)=-y\left(A_{u}^{\prime}+\beta A_{w}\right) \cos (\beta z), \\
& w(x, z)=A_{w}(x) \sin (\beta z),
\end{aligned}
$$

with following boundary conditions of amplitudes

$\left.A_{u}\right|_{x=0}=0,\left.\quad \frac{\mathrm{d} A_{u}}{\mathrm{~d} x}\right|_{x=0}=0,\left.\quad A_{w}\right|_{x=0}=0$,

where $\beta=2 \pi / \lambda$ is the wave number, the symbol "'" denotes $\mathrm{d} / \mathrm{d} x, A_{u}$ and $A_{w}$ are dimensionless amplitudes of $u$ and $w$, respectively.

Then exact solutions of amplitudes are obtained as

$$
\begin{aligned}
A_{u}= & \left(\frac{C_{2}}{2} x-\frac{C_{1}}{4 \beta}\right)\left(\mathrm{e}^{\beta x}-\mathrm{e}^{-\beta x}\right)+\frac{C_{1}}{2} x \mathrm{e}^{-\beta x}, \\
A_{w}= & \frac{1}{2 \beta}\left(\frac{C_{1}}{2}+C_{2}\right)\left(\mathrm{e}^{\beta x}-\mathrm{e}^{-\beta x}\right)+\frac{C_{1}}{2} x \mathrm{e}^{-\beta x} \\
& -\frac{C_{2}}{2} x\left(\mathrm{e}^{\beta x}+\mathrm{e}^{-\beta x}\right),
\end{aligned}
$$

in the inner region, $x \in[0, h]$, where $h$ is the height of nearest-wall region, and $C_{1}$ and $C_{2}$ are positive constants with the specific conditions: $C_{1}=A_{\omega_{y}}, C_{2} \geqslant 2 A_{\omega_{y}}$, and $h=C_{1} /\left(2 C_{2} \beta\right)$. 
Thereby, the induced vertical velocity $v$ is obtained as

$v(x, y, z)=-C_{2} y\left(\mathrm{e}^{\beta x}-\mathrm{e}^{-\beta x}\right) \cos (\beta z)$.

At last, the induced vorticity is obtained as follows

$$
\begin{aligned}
& \omega_{x}(x, y, z)=-C_{2} \beta y\left(\mathrm{e}^{\beta x}-\mathrm{e}^{-\beta x}\right) \sin (\beta z), \\
& \omega_{y}(x, z)=-C_{1} \mathrm{e}^{-\beta x} \sin (\beta z), \\
& \delta \omega_{z}=-C_{2} \beta y\left(\mathrm{e}^{\beta x}+\mathrm{e}^{-\beta x}\right) \cos (\beta z) .
\end{aligned}
$$

Thus, from above vorticity field, $\omega_{x}$ is intrinsically related to introduced $\omega_{y}$, as the first sign law, i.e. $\omega_{x} \cdot \omega_{y} \geqslant 0$ in region of $(x>0, y>0)$ (the upper shear layer) and $\omega_{x} \cdot \omega_{y} \leqslant 0$ in region of $(x>0, y<0)$ (the lower shear layer). Such relationship is irrelevant to induced spanwise vorticity $\delta \omega_{z}$ and original 2-D spanwise vorticity $\Omega_{z}$. From the point of vorticity sign, it clearly shows that $+\left|\omega_{y}\right|$ or $-\left|\omega_{y}\right|$ induces $+\left|\omega_{x}\right|$ or $-\left|\omega_{x}\right|$ in the upper shear layer, respectively, but $-\left|\omega_{x}\right|$ or $+\left|\omega_{x}\right|$ in the lower shear layer, respectively. Such feature is in a good agreement with special distributions of vorticity components at specific signs in mode A in Fig. 4. This further indicates that the positive streamwise vortices in Fig. $4 \mathrm{c}$ are actually originated from the induction of vertical vorticity on the rear surface of the square-section cylinder, i.e. surface BC in Fig. 5a, rather than the result of wavy deformation of primary vortices or Biot-Savart induction, even the vortex-tongue model.

It is also verified that the effect of finite vertical length of the bluff body $D$ on the qualitative analysis above is almost negligible, although the theoretical solutions are obtained based on the local flow region [16]. Near the rear surface, induced $v$ can be expressed approximately by $-y\left(\mathrm{e}^{\beta x}-\mathrm{e}^{-\beta x}\right) \cos (\beta z)$ because of (almost) uniformly distributed $\omega_{y}$ attached on the rear surface, as shown in Fig. 5a and sketched in Fig. 6a. The vertical length scale of $\omega_{y}$ is almost equivalent to $D$. This means that the induced velocity $(u, v, w)$ would be gradually reduced down to zero far away from the rear surface or shear layers due to the gradual disappearance of effect of introduced vertical vortex pair. The typical distributions of $v$ and $w$ can be shown in Fig. 6b, c at different spanwise positions. Therefore, it shows that $\omega_{x}$ is still with a negative sign because of $\partial w / \partial y<0$ and $\partial v / \partial z>0$, for instance, in local region $(y>0,0<z<\lambda / 2)$ and its qualitative distribution is also demonstrated in Fig. 6b, c. Furthermore, the maximal value of $\omega_{x}$ would be appeared in the shear layers where $|v|$ reaches the local maximum and $\partial w / \partial y$ appeares, as shown in Fig. 6b, c, also well agreed with Fig. 4c.

Finally, the effect of induced spanwise vorticity $\delta \omega_{z}$ on original 2-D spanwise vorticity $\Omega_{z}$ or total spanwise vorticity $\omega_{z}$ is also demonstrated as follows. For example in Fig. 6, the sign of $\delta \omega_{z}$ is negative in the upper side of wake center plane (WCP) $(y>0)$ but positive in the lower side of WCP $(y<0)$ at $z=0$, and vice versa at $z=\frac{1}{2} \lambda$, according to Eq. (9c). Such specific variation of $\delta \omega_{z}$ across the span leads to amplitude of $\Omega_{z}$ increased at $z=0$ but decreased at $z=\frac{1}{2} \lambda$. Similar to the effect of fluid compressibility or Kelvin's circulation theorem [14], the reduction of vorticity indicates the expansion of fluid elements with such spanwise vorticity, while the intensification of vorticity denotes the compression of same fluid elements. Similar process is also occurred in the shedding spanwise vortices, just as shown in Fig. 5b with the reduced $\left|\omega_{z}\right|$ and Fig. 5c with the increased $\left|\omega_{z}\right|$ (note that distribution of $\omega_{y}$ in Fig. 5a is just opposite to that in Fig. 6a along the spanwise direction). Consequently, just owning to such undulation of spanwise vorticity along the span, the shear layer and shedding spanwise vortices are wavily varied in a sinusoidal fashion, as well as the wake width.

\section{Conclusion}

In present paper, the origin of streamwise vortices in mode A 3-D instability in the wake of a square cylinder is investigated. Through numerical simulations of flow past a square cylinder at $R e=180$, the sign of streamwise vorticity in mode $\mathrm{A}$ is just opposite to that on lateral surfaces. It is confirmed that the mechanism of streamwise vorticity generated on the surface separated region, even the instability of primary vortex or vortex tongue model, is not primary physical mechanism of such problem.

Furthermore, on the basis of the VIVor theory, the local flow field is analyzed in the immediate neighborhood of rear surface of a square cylinder by introducing vertical vorticity attached on surface. Then the new physical mechanism in the generation of specific streamwise vortices is identified. Its formation process is presented as follows: (1) the vertical vorticity on the rear surface is firstly generated by the 3-D instaiblity near the cylinder, similar to streamwise vorticity near the wavy separation line; (2) then through the physical mechanism of vortex-induced vortex, the streamwise vorticity at a specific sign is induced by the above vertical vorticity just behind the cylinder, convected into and accumulated in the shear layers; (3) finally the streamwise vorticies are formed and shed alternatively with the spanwise vortices.

On the other hand, the induced spanwise vorticity leads to the original 2-D spanwise vorticity increased or decreased across the span. Consequently, the spanwise vortices, as well as the shear layers and wake width, are wavily along the span.

In future, the origin of streamwise vortices in mode A for the circular-section cylinder would be investigated through direct numerical simulations. 
Acknowledgements This work was financially supported by the Strategic Priority Research Program of Chinese Academy of Science (Grant XDB22030101).

\section{References}

1. Wu, Z.B., Ling, G.C.: Numerical study on the mechanism for threedimensional evolution of vortex and the structural features in the wake behind a circular cylinder. Acta Mech. Sin. 9, 264-275 (1993)

2. Brede, M., Eckelmann, H., Rockwell, D.: On secondary vortices in the cylinder wake. Phys. Fluids 8, 2117-2124 (1996)

3. Williamson, C.H.K.: Three-dimensional wake transition. J. Fluid Mech. 328, 345-407 (1996)

4. Williamson, C.H.K.: Vortex dynamics in the cylinder wake. Annu. Rev. Fluid Mech. 28, 477-539 (1996)

5. Barkley, D., Henderson, R.D.: Three dimensional Floquet stability analysis of the wake of a circular cylinder. J. Fluid Mech. 322, 215-241 (1996)

6. Henderson, R.D.: Nonlinear dynamics and pattern formation in turbulent wake transition. J. Fluid Mech. 352, 65-112 (1997)

7. Ling, G.C., Chang, Y.: Three-dimensional stability analysis of the periodic wake behind a circular cylinder by low-dimensional Galerkin method. Acta Mech. Sin. 15, 652-660 (1999)

8. Luo, S.C., Tong, X.H., Khoo, B.C.: Transition phenomena in the wake of a square cylinder. J. Fluids Struct. 23, 227-248 (2007)

9. Jiang, H., Cheng, L., Draper, S., et al.: Three-dimensional direct numerical simulation of wake transitions of a circular cylinder. J. Fluid Mech. 801, 353-391 (2016)

10. Agbaglah, G., Mavriplis, C.: Computational analysis of physical mechanisms at the onset of three-dimensionality in the wake of a square cylinder. J. Fluid Mech.833, 631-647 (2017)
11. Jiang, H.Y., Cheng, L., An, H.W.: Three-dimensional wake transition of a square cylinder. J. Fluid Mech. 842, 102-127 (2018)

12. Yokoi, Y., Kamemoto, K.: Initial stage of a three-dimensional vortex structure existing in a two-dimensional boundary layer separation flow (Observation of laminar boundary layer separation over a circular cylinder by flow visualization). JSME Int. J. Ser. II, 189-195 (1992)

13. Yokoi, Y., Kamemoto, K.: Initial stage of a three-dimensional vortex structure existing in a two-dimensional boundary layer separation flow (Visual observation of laminar boundary layer separation over a circular cylinder from the side of a separated region). JSME Int. J. Ser. B 36, 201-206 (1993)

14. Green, S.I.: Fluid Votices. Kluwer Academic Publishers, Norwell (1995)

15. Lin, L.M., Zhong, X.F., Wu, Y.X.: Effect of perforation on flow past a conic cylinder at $R e=100$ : wavy vortex and sign laws. Acta Mech. Sin. (2018). https://doi.org/10.1007/s10409-018-0758-z,. (in press)

16. Lin, L.M., Shi, S.Y., Zhong, X.F., et al.: Mechanism of wavy vortex and sign laws in flow past a bluff body: vortex-induced vortex, Acta Mech. Sin. (2018). https://doi.org/10.1007/s10409018-0793-9. (in press)

17. Robichaux, J., Balachandar, S., Vanka, S.P.: Three-dimensional Floquet instability of the wake of square cylinder. Phys. Fluids 11, 560-578 (1999)

18. Karniadakis, G.E., Israeli, M., Orszag, S.L.: High-order splitting methods for the incompressible Navier-Stokes equatcons. J. Comput. Phys. 97, 414-443 (1991)

19. Lin, L.M.: Wake dynamics and forces in the flow around the square-section cylinder with a geometric disturbance, [Ph.D Thesis], Institute of Mechanics, Chinese Academy of Sciences, (2007)

20. Luo, S.C., Chew, Y.T., Ng, Y.T.: Characteristics of square cylinder wake transition flows. Phys. Fluids 15, 2549-2559 (2003) 